\title{
Complete Second Branchial Fistula: A Study of Four Cases
}

KJ Shinde

\begin{abstract}
Objective: Complete branchial cleft fistulae are rare anomalies of branchial cleft apparatus. Incomplete fistulae are more common to occur which may not be symptomatic at all and neglected as it do not give rise to recurrent infection. While complete brancial fistula gives persistent symptom of discharge from the fistula. This is a report of four cases of complete branchial fistulae which were diagnosed and treated surgically in a span of 2 years in the Department of ENT, Head and Neck Surgery, Rural Medical College and Pravara Rural Hospital, Loni. The main objective of this report is the use of fistulogram for the diagnosis and complete excision of fistula including the both inner and outer ends of fistulae.
\end{abstract}

Case report: Four cases were reported in the age group between 11 and 22 years who presented with an intermittent mucoid discharge from an external opening in the neck since birth with right to left ratio being $4: 1$ and male:female ratio being $1: 1$ and were diagnosed to have a second branchial cleft fistula. A preoperative fistulogram revealed the tracts up to the tonsillar fossa with connection over the skin surface in the neck. Complete excision of the tracts were done by a two-step or step ladder neck incision.

Conclusion: Though second branchial fistulae are common, complete fistulae are rare with its complete excision. The report also stresses on the importance of a preoperative fistulogram for the preoperative diagnosis and correct complete surgical treatment.

Keywords: Branchial fistula, Complete branchial fistula, Branchial cleft, Management of complete branchial fistula.

How to cite this article: Shinde KJ. Complete Second Branchial Fistula: A Study of Four Cases. Int J Head Neck Surg 2013;4(3):129-132.

\section{Source of support: Nil}

Conflict of interest: None declared

\section{INTRODUCTION}

Congenital cysts, sinuses and fistulae must be considered in the diagnosis of head and neck masses in children and adults. Anomalies of the second branchial fistula account for $90 \%$ of the developmental abnormalities of the branchial apparatus. Branchial fistula usually present in childhood as a continuously discharging defect along the anterior border of sternocleidomastoid muscle at the junction of middle and lower $1 / 3$ rd of neck. This extends up through neck to end its opening in tonsillar fossa/pillars. This is a report of four cases of complete second branchial cleft fistulae.

\section{MATERIALS AND METHODS}

These cases presented with symptoms in Pravara Rural Hospital and further case studies were carried out in Outpatient
Department of Otorhinolaryngology, Head and Neck Surgery, Pravara Rural Hospital, Loni, Ahmednagar, in a span of 2 years from 2010 to 2012.

\section{CASE STUDIES OF FOUR CASES}

Four cases were reported to ENT OPD who were diagnosed as complete second branchial cleft fistulae in the age group between 11 and 22 years with male:female ratio being 1:1 and right: left side ratio being $3: 1$. All these patients presented to outpatient clinic with history of small discharging opening in the lower part of the neck since birth. There was intermittent, yellowish white discharge from the opening. Discharge from sinus opening was associated with fever in one of the case. On examination, a small opening was seen on neck along the anterior border of the sternomastoid at junction of middle and lower 1/3rd with scanty mucous discharge coming out on pressure. In one of the case, margins of the sinus opening were inflamed with active mucopurulent discharge (Fig. 1).

On examination of throat, the oropharynx did not reveal any visible opening in the pharynx. On the basis of clinical examination and symptoms, they were diagnosed as comlete branchial fistula. A fistulogram showed the tract extending from the neck until the tonsillar region in the pharynx. Dye was seen entering the lateral wall of the oropharynx in the tonsillar region (PA and lateral view of soft tissue neck) (Fig. 2).

After necessary routine investigations, excision of the complete fistulous tract was carried out under general anesthesia. Aim of surgery was to perform complete excision

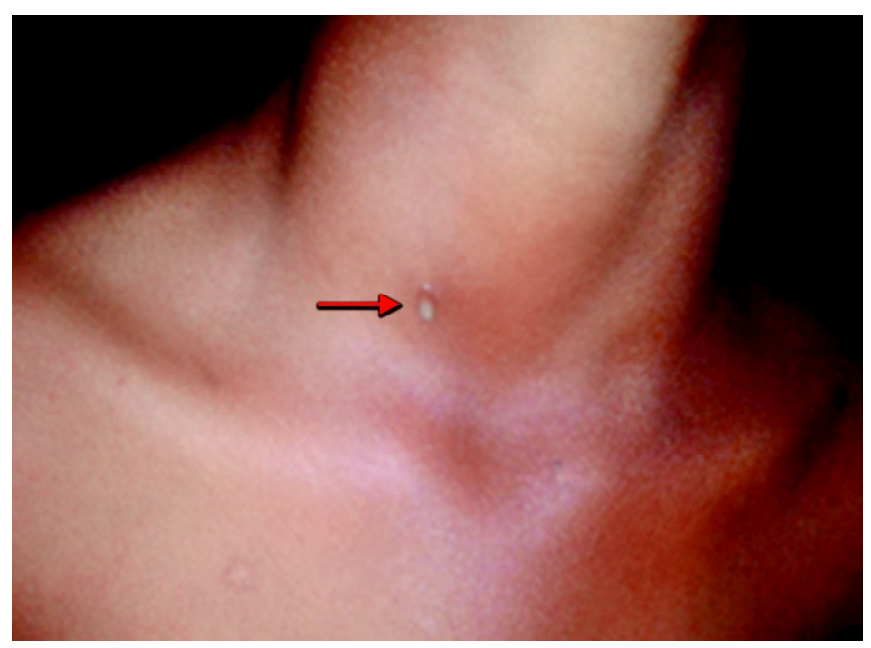

Fig. 1: Sinus opening with active mucopurulent discharge 
of fistulous tract alongwith tonsillectomy. Step ladder dissection was performed starting initially in the neck with combined approach via transcervical and transoral approach under general anesthesia.

Initially, circumscribed skin incision was taken around fistulous opening. The fistulous tract was then separated from skin and fascia over it. Second incision (step ladder dissection) was taken at the level of hyoid bone and fistulous tract was pulled through second incision to carry out further dissection toward oropharynx. Inspection of oropharynx was then done. Confirmed attachment with tonsilar area and tonsil with fistulous tract was excised along with it (Fig. 3).

After complete excision of fistula, alongwith tonsil was almost 14 to $15 \mathrm{~cm}$ inlength. It was possible in all cases to remove fistulous tract with tonsil in total (Fig. 4).

Histopathological examination confirmed the keratinized squamous epithelial lining of the tract with clusters of mucous secreting glands at places. No recurrence of symptoms is seen with 6 months of follow-up.

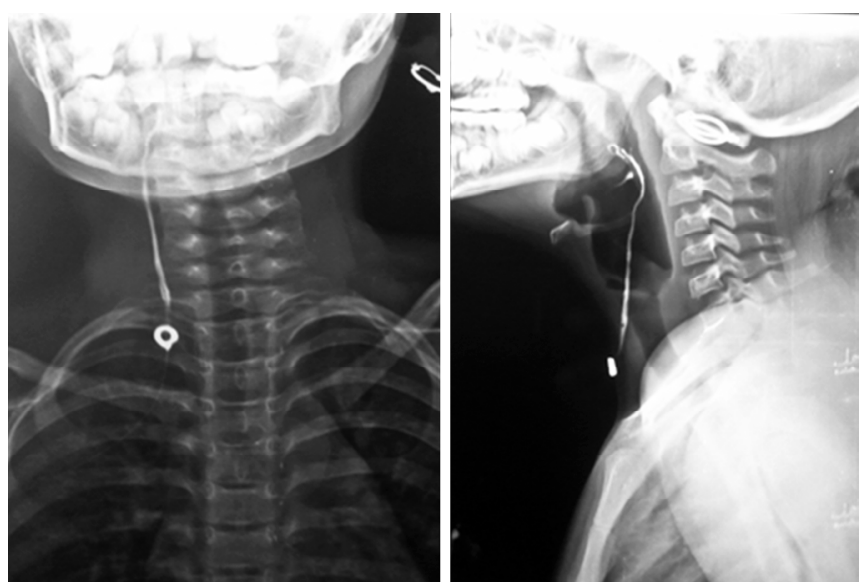

Fig. 2: Fistulogram showing the tract extending in neck up to the tonsillar region

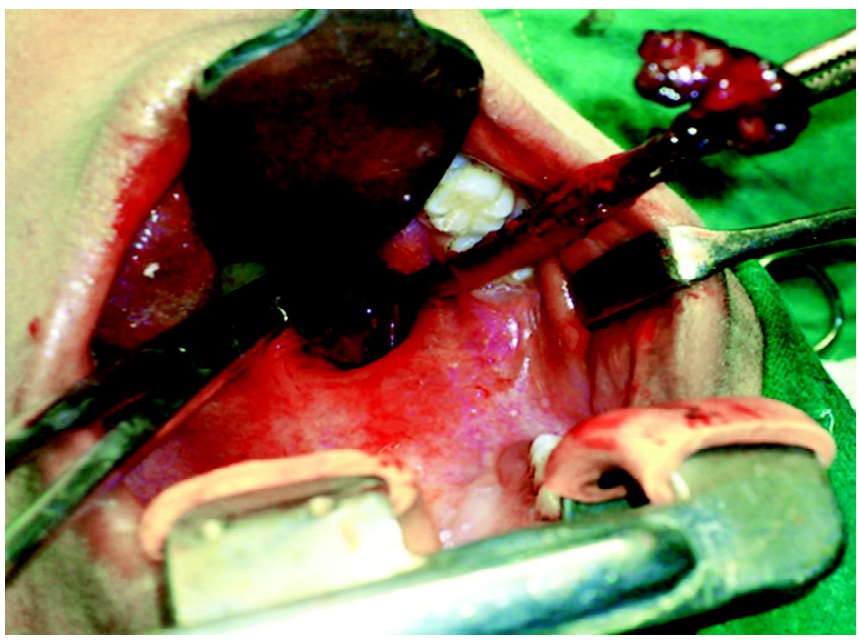

Fig. 3: Tonsil with fistulous tract in the process of excision

\section{DISCUSSION}

Although the branchial apparatus was first described by von Baer, anomalies in its development were credited by von Ascheron. ${ }^{1}$ Branchial arches and their corresponding pouches develop from mesodermal condensations in the side wall of the embryonic pharynx. Branchial fistulae are uncommon anomalies of embryonic development of branchial apparatus. Second branchial arch and pouch anomalies are common anomalies of branchial apparatus. ${ }^{2}$ During embryonic development, the second arch grows caudally, envelop the third, fourth and sixth arches and form the cervical sinus by fusing with the skin caudal to these arches. The edges of cervical sinus fuse and the ectoderm within the fused tube disappears. Persistence of ectoderm gives rise to branchial cyst. The branchial fistula results from the breakdown of the endoderm, usually in the second pouch. A persistent fistula of the second branchial cleft and pouch pass from the external opening in the mid or lower neck in the line of the anterior border of the sternocleidomastoid muscle, deep to platysma along the carotid sheath, then pass medially deep between the internal and external carotid arteries after crossing over the glossopharyngeal nerve and hypoglossal nerve. Finally, it opens internally in the tonsillar fossa usually on the anterior face of the upper half of the posterior pillar of the fauces or in the intratonsillar cleft. ${ }^{3}$ Most of times, it is a simple sinus opening, that extends up the neck for a variable distance. Complete branchial fistula with internal opening into tonsillar region is rare. Although branchial fistulas may occur in any age group, commonly patients present to clinician in first and second decades of life. $^{4}$

Anomalies of the second branchial cleft account for $90 \%$ of the developmental abnormalities of the branchial apparatus. ${ }^{5}$ However, complete second arch fistulae are rare and comprise $2 \%$ of all branchial anomalies. The fistula is

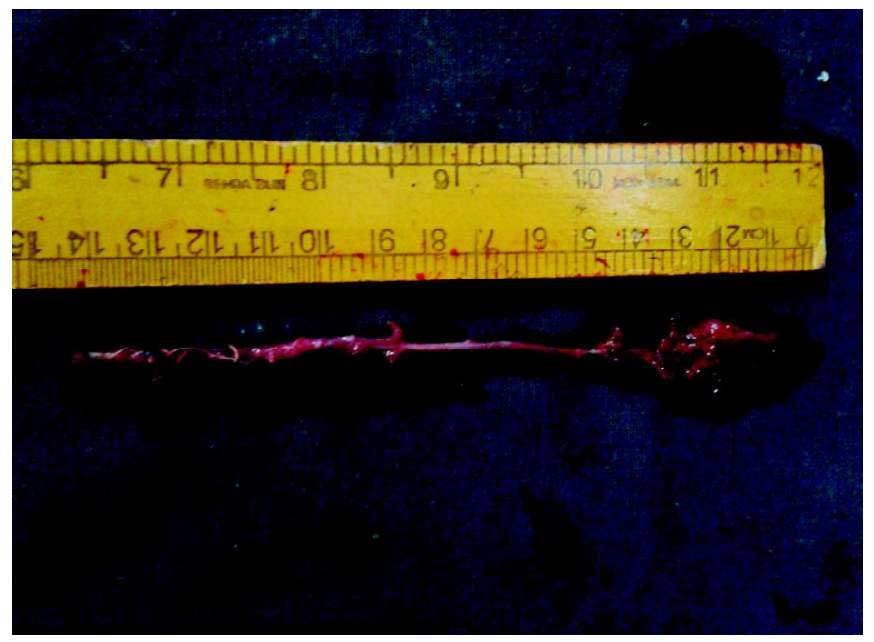

Fig. 4: Tonsil with excised fistulous tract 
more commonly seen in males $(60 \%)$ and can range from 1 to 8 cmin length. ${ }^{6}$ Two to ten percent of themcan be bilateral. When unilateral, $70 \%$ of them occur on the right side. Thirtynine percent are complete fistulae, $50 \%$ are external draining sinuses and $11 \%$ have internal opening alone. ${ }^{7}$ Thirty-five percent of the patients with complete fistula can have a family history of branchial anomalies. ${ }^{7}$ Branchial cysts are more common $(80.8 \%)$ than branchial fistulae. ${ }^{8}$

Anatomically, a typical second cleft fistula has its external opening at the anterior border of sternomastoid at the junction of middle and lower 1/3rd. Second arch anomalies are classified into four types: Type I lesions lie anterior to the sternocleidomastoid muscle (SCM) and do not come in contact with the carotid sheath. Type II lesions are the most common and pass deep to the SCM and either anterior or posterior to the carotid sheath. Type III lesions pass between the internal and external carotid arteries and are adjacent to the pharynx. Type IV lesions lie medial to the carotid sheath close to the pharynx adjacent to the tonsillar fossa. ${ }^{9}$ Our cases had the type II pattern. In the upper part, the tract may end in the upper half of the posterior tonsillar pillar, the supratonsillar fossa or directly onto the tonsillar surface. ${ }^{7}$ The fistulae are almost always present at birth with a small pinpoint external opening which may go unnoticed. Some patients may also have conductive or sensory neural deafness as well as other anomalies of the first and second arch derivatives. ${ }^{7}$

Symptoms consist of intermittent or continuous mucous discharge and recurrent attacks of inflammation following an attack of upper respiratory tract infection. Frank cellulitis or abscess formation may occur requiring an incision and drainage. The external opening may be seen to move upwards with deglutition. Probing the tract may sometimes produce symptoms of cough, palpitation, pallor and vomiting because of the tract's proximity to the vagus nerve. ${ }^{7}$ Pathologically, the fistula lining consists of squamous epithelium.

A fistulogram may be obtained to confirm the clinical diagnosis and is useful to show the length and the location of the tract and possible presence of associated cyst. ${ }^{7}$ Radiographs reveal a smoothly marginated tract of variable width following the anatomical path as described earlier. In contrast, the walls of a tuberculous fistula are very irregular. ${ }^{7}$ According to another study; a routine preoperative fistulogram may not be required in all cases. ${ }^{8}$

Surgery is usually not indicated if the fistula is asymptomatic. However, most are symptomatic and the surgical excision is carried out to avoid the risk of recurrent infection and for cosmetic reasons. Sclerosing agents are seldom used today due to the associated inflammatory reaction and the risk of necrosis with perforation into the pharynx. ${ }^{5}$
Incisions for the excision could be hockey stick type ${ }^{10}$ or step ladder incision (Bailey). A similar technique involving two separate transverse incisions is now recommended for the removal of the branchial fistulae. ${ }^{5}$ We have done surgical excision of all five cases by starting with elliptical excision of fistula operation. Dissection of track can be helped by injection of dye into the opening before start of procedures. As all are second branchial fistulae, they required further skin incision to avoid injury to carotid vessel. Excision of tonsillar fossa opening to get continuous removal of tract. Another variation, pull through, branchial fistulectomy has been described by Talaat in $1992 .{ }^{5}$ In this technique, the infrahyoid portion of the fistula is dissected through one or two (stepped) neck incisions. Dissection of the parapharyngeal segment is done via the mouth and is continued to the level of the hyoid bone. Tonsillectomy is then performed; after which the fistula is withdrawn through the mouth. The stripping method was described by Taylor and Bicknell in 1977. But, this has not been widely used due to the greater risk of damage to the adjacent structures. $^{7}$

Complications of the surgery include recurrence, which could be $3 \%$ in fresh cases to up to $20 \%$ in second surgical attempts. Other complications include secondary infection, injury to facial, hypoglossal, glossopharyngeal, spinal accessory nerves, injury to internal jugular vein, bad scar and hematoma formation. ${ }^{10}$ Till now with combined transcervical and transoral approach, there is no recurrence reported in the English literature.

\section{CONCLUSION}

Complete branchial fistula arising from second branchial cleft is rare. It is not possible to excise the complete branchial fistula totally with transcervical approach only. This is a case series of complete branchial fistulae, which were managed through combined approach using transcervical route and transoral route. Complete branchial fistulae are better managed by otolaryngologists who are capable of performing the combined approach.

\section{REFERENCES}

1. De PR, Mikhail T. A combined approach excision of branchial fistula. J Laryngol Otol 1995;109:999-1000.

2. Ford GR, Balakrishnan A, Evans IN, et al. Branchial cleft and pouch anomalies. J Laryngol Otol 1992;106:137-43.

3. Talaat M. Pull-through branchial fistulectomy: Technique for the otolaryngologist. Ann Otol Rhino Laryngol 1992;101:501-02.

4. Ang AH, Pang KP, Tan LK. Complete branchial fistula. Case report and review of the literature. Ann Otol Rhinol Laryngol 2001;110:1077-79.

5. Ismail $\mathrm{Y}$, Ozcan $\mathrm{C}$, Nuri $\mathrm{O}$, Fatih $\mathrm{B}$, Beyhan D. Complete fistula of the second branchial cleft: Case report of catheter aided total excision. Int J Ped Otorinolaryngol 2004;68:1109-13. 
6. Broadford G, John M, Mike B, Sugki C. Aberrant second branchial cleft fistula. Int J Ped Otorinolaryngol 1998;46: 103-07.

7. Burton MG. Second branchial cleft cyst and fistula. Am J Radiol 1980 May;134:1067-69.

8. Kamal NR, Simi R, Dheeraj P, Joginder SG, Samar Pal Singh Y. Second branchial cleft fistula. Is fistulogram necessary for total excision. Int J Ped Otorinolaryngol 2006;70:1027-30.

9. Stephanie P, Acierno, John HT, Waldhausen. Congenital cervical cysts, sinuses and fistulae. Otolaryngol Clin North Am 2007;40: 161-76.
10. Francisco C, Agaton B, Cosmay GE. Diagnosis and treatment of branchial cleft cysts and fistulae. A retrospective study of 183 patients. Int J Oral Maxillofac Surg 1996;25: 449-52.

\section{ABOUT THE AUTHOR}

\section{KJ Shinde}

Professor and Head, Department of ENT, Rural Medical College Loni, Ahmednagar, Maharashtra, India, e-mail: Kiranpmt@yahoo.com 